\title{
Evaluation of Neurodegeneration in a Mouse Model of Infantile Batten Disease by Magnetic Resonance Imaging and Magnetic Resonance Spectroscopy
}

\author{
Jeeva Munasinghe ${ }^{a}$ Zhongjian Zhang $^{b}$ Eryan Kong ${ }^{b}$ Alison Heffer ${ }^{b}$ \\ Anil B. Mukherjee ${ }^{b}$ \\ a In Vivo NMR Center (HNQ2-3), National Institute of Neurological Disorders and Stroke, National Institutes of \\ Health, and ${ }^{b}$ Program on Developmental Endocrinology and Genetics, Eunice Kennedy Shriver National Institute \\ of Child Health and Human Development, National Institutes of Health, Bethesda, Md., USA
}

\section{Key Words \\ Neurodegeneration - Neuronal ceroid lipofuscinosis • Infantile neuronal ceroid lipofuscinosis - Infantile batten disease $\cdot$ Magnetic resonance imaging $\cdot$ Magnetic resonance spectroscopy}

\begin{abstract}
Neuronal ceroid lipofuscinoses (NCLs) represent a group of common hereditary childhood neurodegenerative storage disorders that have no effective treatment. Mutations in eight different genes cause various forms of NCLs. Infantile $\mathrm{NCL}$ (INCL), the most lethal disease, is caused by inactivating mutations in the palmitoyl-protein thioesterase-1 (PPT1) gene. The availability of Ppt1-knockout (Ppt1-KO) mice, which recapitulate virtually all clinical and pathological features of $I N C L$, provides an opportunity to test the effectiveness of novel therapeutic strategies in vivo. However, such studies will require noninvasive methods that can be used to perform serial evaluations of the same animal receiving an experimental therapy. Thus, the development of noninvasive method(s) of evaluation is urgently needed. Here, we report our evaluation of the progression of neurodegeneration in Ppt1-KO mice starting at 3 months of age by MRI and MR
\end{abstract}

(C) 2012 S. Karger AG, Basel

$1660-2854 / 12 / 0094-0159 \$ 38.00 / 0$

Fax +41613061234

E-Mail karger@karger.ch

www.karger.com
Accessible online at:

www.karger.com/ndd spectroscopy (MRS) and repeating these tests using the same mice at 4, 5 and 6 months of age. Our results showed progressive cerebral atrophy, which was associated with histological loss of neuronal content and increase in astroglia. Remarkably, while the brain volumes in Ppt1-KO mice progressively declined with advancing age, the MRS signals, which were significantly lower than those of their wild-type littermates, remained virtually unchanged from 3 to 6 months of age. In addition, our results also showed an abnormality in cerebral blood flow in these mice, which showed progression with age. Our findings provide methods to serially examine the brains of mouse models of neurodegenerative diseases (e.g. Ppt1-KO mice) using noninvasive and nonlethal procedures such as MRI and MRS. These methods may be useful in studies to understand the progression of neuropathology in animal models of neurodegenerative diseases as they allow repeated evaluations of the same animal in which experimental therapies are tested.

Copyright $\odot 2012$ S. Karger AG, Basel

J.M., Z.Z., E.K. and A.H. equally contributed to this paper.

Anil B. Mukherjee, $\mathrm{MD}, \mathrm{PhD}$

NIH, Building 10, Room 9D42

10 Center Drive

Bethesda, MD 20892-1830 (USA)

Tel. +1 301496 7213, E-Mail mukherja@exchange.nih.gov 


\section{Introduction}

Neurodegeneration is a devastating manifestation in the majority of more than 40 lysosomal storage disorders [reviewed in 1,2] and the development of effective treatment for these disorders remains challenging. The neuronal ceroid lipofuscinoses (NCLs), also known as Batten disease, represent a group of common (1 in 12,500 births) neurodegenerative lysosomal storage disorders that primarily affect children [3-11]. To date, mutations in eight different genes have been reported to cause various types of NCLs $[9,10]$. The infantile form of NCL (INCL), also known as infantile Batten disease, is a rapidly progressive, uniformly fatal neurodegenerative storage disorder without any effective treatment [6]. Children with INCL are normal at birth, but by 11-18 months of age they develop psychomotor retardation. By 2 years of age, these children are completely blind due to retinal degeneration and by 4 years of age they fail to manifest any brain activity. These children remain in a vegetative state for 6-8 more years before death. These grim facts underscore the necessity for developing effective therapeutic strategies.

Palmitoylation (also known as S-acylation) is a posttranslational modification in which a 16 -carbon fatty acid, palmitate, is attached to specific cysteine residue(s) in polypeptides via thioester linkage [12-15]. To date, more than 100 proteins have been reported to undergo palmitoylation [12] and this list continues to grow. A large number of these proteins are represented in the central nervous system [12-14]. While palmitoylation is required for the function of many proteins, depalmitoylation (removal of palmitate) is also critical for these proteins to recycle or be degraded by lysosomal proteases [16]. Currently, two enzymes are known to catalyze depalmitoylation of S-acylated proteins. Among these, acylprotein thioesterase-1 [17] and acyl-protein thioesterase-2 [18] are cytosolic, while palmitoyl-protein thioesterase-1 (PPT1) [19, 20] and PPT2 [21] are lysosomal enzymes [22, 23]. The importance of dynamic palmitoylation (palmitoylation/depalmitoylation) of S-acylated proteins is well recognized, especially in the central nervous system $[24,25]$ as mutations in a gene encoding a depalmitoylating enzyme, PPT1, cause a devastating neurodegenerative storage disorder, INCL [26]. PPT1 catalyzes the cleavage of thioester linkage in palmitoylated (S-acylated) proteins, which is required for their degradation by lysosomal proteases $[6,16]$. Thus, it has been suggested that lack of PPT1 activity leads to abnormal lysosomal accumulation of undegraded lipid-modified proteins (constituent of ceroid), causing INCL pathogenesis.
However, the precise molecular mechanism(s) underlying INCL pathogenesis remains poorly understood. Consequently, the development of rational and effective therapies remains challenging.

Ppt1-knockout (Ppt1-KO) mice [27] recapitulate virtually all clinical and pathological features of human INCL, providing a reliable animal model for this disease [28] which can be used to test novel therapeutic approaches. Although neuropathology of these mice has been studied extensively, the progression of neurodegeneration in live Ppt1-KO mice using noninvasive methods, to our knowledge, has not been documented. Clearly, reliable noninvasive methods are urgently needed to repeatedly test the effects of potential therapeutic interventions in vivo. The Ppt1-KO mice provide a reliable model for obtaining the proof of principle that such therapeutic strategies are effective. Towards this goal, we sought to determine whether the use of MRI and MR spectroscopic (MRS) analyses of the brain are useful methods for repeated evaluation of disease progression in Ppt1-KO mice. Here, we report that MRI and MRS are useful techniques that can be applied repeatedly to evaluate the progression of neurodegenerative changes in the brain of live Ppt1-KO mice. Our results suggest that these techniques may be useful for delineating the effectiveness of novel therapeutic approaches for INCL.

\section{Animals and Methods}

\section{Animals}

Ppt1-KO mice were generated by targeted disruption of the last exon in the Ppt1 gene in embryonic stem cells as previously reported [27]. These mice were subsequently backcrossed for 10 generations with wild-type (WT) C57BL/6 mice in order to obtain congenic C57 background and a breeding pair was kindly given to us by Dr. Mark Sands to start our colony at the NIH. An animal study protocol to conduct the MRI/MRS studies was approved by the institutional (NICHD, NIH) animal care and use committee. Age- and sex-matched Ppt1-KO mice and their WT littermates were used for these studies.

Brain MRI/MRS of Ppt1-KO Mice and Their WT Littermates

Ppt1-KO mice $(\mathrm{n}=5)$ and their WT littermates $(\mathrm{n}=4-5)$, were anesthetized with $1.5 \%$ isoflurane and allowed to breathe spontaneously. Since the mice were not mechanically ventilated, $\mathrm{pCO}_{2}$ was not monitored. The dorsal tail veins of mice were positioned in a stereotaxic holder for MRI/MRS and catheterized in order to administer ultrasmall iron oxide particles (USPIO) as contrast. The body core temperature was maintained at $37^{\circ} \mathrm{C}$ using a circulating water pad and monitored throughout the procedure. MRI was performed on a 7-Tesla 21-cm horizontal scanner (Bruker Avance, Billerica, Mass., USA) with the brain centered in a 72 volume (transmit) $/ 25 \mathrm{~mm}$ surface (receive) radio frequency coil ensemble to record the MR signal. 
MR images, acquired in three mutually orthogonal directions, were used as a scout to obtain qualitative and quantitative information of the brain tissue of the normal and Ppt1-KO mice. First, specified via the pilot scans, multiple slice fast-spin echo [spinspin relaxation $\left(\mathrm{T}_{2}\right)$-weighted] axial images, encompassing the whole brain [number of averages $=8$, echo train length $=8$, repetition time $(\mathrm{TR})=3,000 \mathrm{~ms}$, echo time $(\mathrm{TE})=10 \mathrm{~ms}$, in-plane resolution $=75 \mu \mathrm{m}$, slice thickness $=1 \mathrm{~mm}$ ] were acquired to calculate the brain volume of these mice at 3, 4, 5 and 6 months of age. Subsequently, studies were performed using different cohorts of 3and 6-month-old mice. A set of gradient echo [spin lattice relaxation time $\left(\mathrm{T}_{1}\right)$-weighted] images $(\mathrm{TR} / \mathrm{TE}=300 / 6 \mathrm{~ms})$, at identical locations and resolution as in images described above, was acquired before and $5 \mathrm{~min}$ after the infusion of USPIO $(20 \mathrm{mg} \mathrm{Fe} / \mathrm{kg}$ body weight; BioPhysics Assay Labs Inc., Worcester, Mass., USA).

Five, $1-\mathrm{mm}$ thick, spin-spin $\left(\mathrm{T}_{2}\right)$ relaxation time-weighted $(\mathrm{TE}=10 \mathrm{~ms}, \mathrm{TR}=3,000 \mathrm{~ms}, 16$ echos, in-plane resolution $=150$ $\mu \mathrm{m})$ and diffusion-weighted $(\mathrm{DW})[\mathrm{TE}=30 \mathrm{~ms}, \mathrm{TR}=3,000 \mathrm{~ms}$, time between gradient sensitizing gradients $(\Delta)=20 \mathrm{~ms}, 4$ gradient sensitizing-b-values from $0-1,500 \mathrm{~mm} / \mathrm{s}, 3$ orthogonal directions, $10 \mathrm{~min} /$ per scan] axial images were acquired. These five slices were positioned with $2-\mathrm{mm}$ gaps between each slice in the anterior-posterior direction; the first slice was approximately $6 \mathrm{~mm}$ posterior to the bregma.

MRS was performed on a localized volume element (voxel $2.5 \mathrm{~mm}^{3}$ ) that was positioned approximately $1 \mathrm{~mm}$ anterior to the bregma, encompassing the major part of the frontal cortex with some overlap in the hippocampus and cerebrospinal fluid. First, using the MR spectra of the dominant water peak, acquired using a point-resolved spectroscopy pulse sequence $(\mathrm{TR} / \mathrm{TE}=1,500 / 16$ $\mathrm{ms}$, number of averages $=44,000$ data points), the magnetic field within this voxel was optimized to be as homogeneous as possible ( $>5 \mathrm{~min}$ ). In order to achieve similar metabolite peak resolutions, the magnetic field optimizations were performed until the average half-height line width of the water resonance peak was 22.2 $\pm 1.9 \mathrm{~Hz}$. Subsequently, the same point-resolved spectroscopy sequence was repeated (number of averages $=512$, total scan time $=25 \mathrm{~min}$ ) after incorporating the appropriate pulse sequence to suppress the dominant water peak within that chosen voxel. The total imaging time taken to complete all of the procedures mentioned above was approximately $1.5 \mathrm{~h}$ per mouse.

The brain volumes and areas under the MR spectral peaks were calculated using MRI scanner display software (Bruker Biospin Inc., Billerica, Mass., USA). The areas encompassing the brain, in each slice, were evaluated by drawing regions of interest and then multiplying by the slice thickness $(1 \mathrm{~mm})$ to calculate the volume. The water-suppressed MR data were processed and spectral chemical shifts were assigned with respect to the creatine peak $(3.05$ ppm). Analysis of the complex spectra were confined to those peaks that corresponded to creatine, choline (3.22 ppm), N-acetyl aspartate (NAA, $2.06 \mathrm{ppm}$ ) and myoinositol (3.6 ppm), and were evaluated by deconvoluting those peaks to a Lorenztian line shape and calculating the areas under those peaks which were proportional to the metabolite concentrations within the chosen voxel.

Quantitative $\left(\mathrm{T}_{2}\right)$ and apparent diffusion coefficient (ADC) maps, from $T_{2}$ and DW images, and relative cerebral blood volumes (rCBV) for regions of interest in selected anatomical areas of the cortex (frontal, parietal) and hippocampus were calculated using programs written in MATLAB (Mathworks Inc., Natick, Mass., USA). In order to present the results, region of interest re- sults from the left and right hemispheres and each region (frontal, parietal) were averaged for each slice and subsequently averaged over all slices.

\section{Golgi-Cox Staining}

Brains from 3-, 4-, 5- and 6-month-old Ppt1-KO mouse and those of their WT littermates were used for Golgi impregnation using the EZ Golgi Kit (Cornell University). The EZ Golgi Kit (solution $\mathrm{A}$ and $\mathrm{B}$ ) was stored in a dark place at $4^{\circ} \mathrm{C}$ and all incubations were performed without exposure to light. Briefly, freshly extracted brains from the skull were immersed in solution A (30 $\mathrm{ml} /$ brain) for 7-10 days at room temperature. Following incubation in solution $\mathrm{A}$, brains were transferred to $30 \%$ sucrose solution $\left(30 \mathrm{ml} /\right.$ brain), and incubated for a minimum of 2 days at $4{ }^{\circ} \mathrm{C}$; after the initial incubation for $10 \mathrm{~h}$, the sucrose solution was changed once. The brain samples were then embedded in 3\% low-melt agarose solution and $150-\mu \mathrm{m}$ thick serial sections were prepared at room temperature using a Pelco 1050 vibratome. The sections were floated on a $30 \%$ sucrose water bath at room temperature and immediately mounted on $0.3 \%$ gelatin-treated glass slides. The tissue sections on slides were dehydrated at room temperature for 3-5 min and then brushed with 50\% sucrose solution to prevent cracking. The tissue sections on slides were then allowed to air dry for at least $48 \mathrm{~h}$. Slides were then dipped 3 times (10 min each) in double distilled water with gentle shaking, transferred to developing solution for 5-10 min and rinsed three times (10 min each) in double distilled water. This was followed by passing them through a series of increasing concentrations of ethanol for dehydration, and then they were cleared 3 times ( 5 min each) with Histoclear and mounted with coverslip using DPX mounting medium.

\section{Immunohistochemistry}

The mouse brain tissues were fixed in 3.7\% paraformaldehyde, embedded in paraffin and processed for histological analyses. The brain sections were incubated with rabbit anti-glial fibrillary acidic protein antibody (1:200; Affinity Bioreagents, Rockford, Ill., USA) at $4^{\circ} \mathrm{C}$ overnight. After rinsing three times with $1 \times$ PBS, the sections were incubated with polyclonal anti-rabbit biotinylated secondary antibody (1:500; Vector Laboratories, Burlingame, Calif., USA) for $1 \mathrm{~h}$ at room temperature. The sections were washed in $1 \times$ PBS and subsequently incubated with prepared ABC complex according to the manufacturer's protocol (Vector Laboratories). The immunostained sections were then analyzed using a Spot RT3 microscope.

Statistical Analysis

Statistical analyses of data were performed using two-way ANAOVA for Ppt1-KO versus WT mice and $\mathrm{p}<0.01$ was considered significant.

\section{Results}

\section{Evaluation of Age-Dependent Progressive Decline in Brain Volume in Ppt1-KO Mice by MRI}

The progression of neurodegeneration in INCL patients is rapid and initially involves the cerebral cortex. Ppt1-KO mice [27] recapitulate virtually all characteristic 

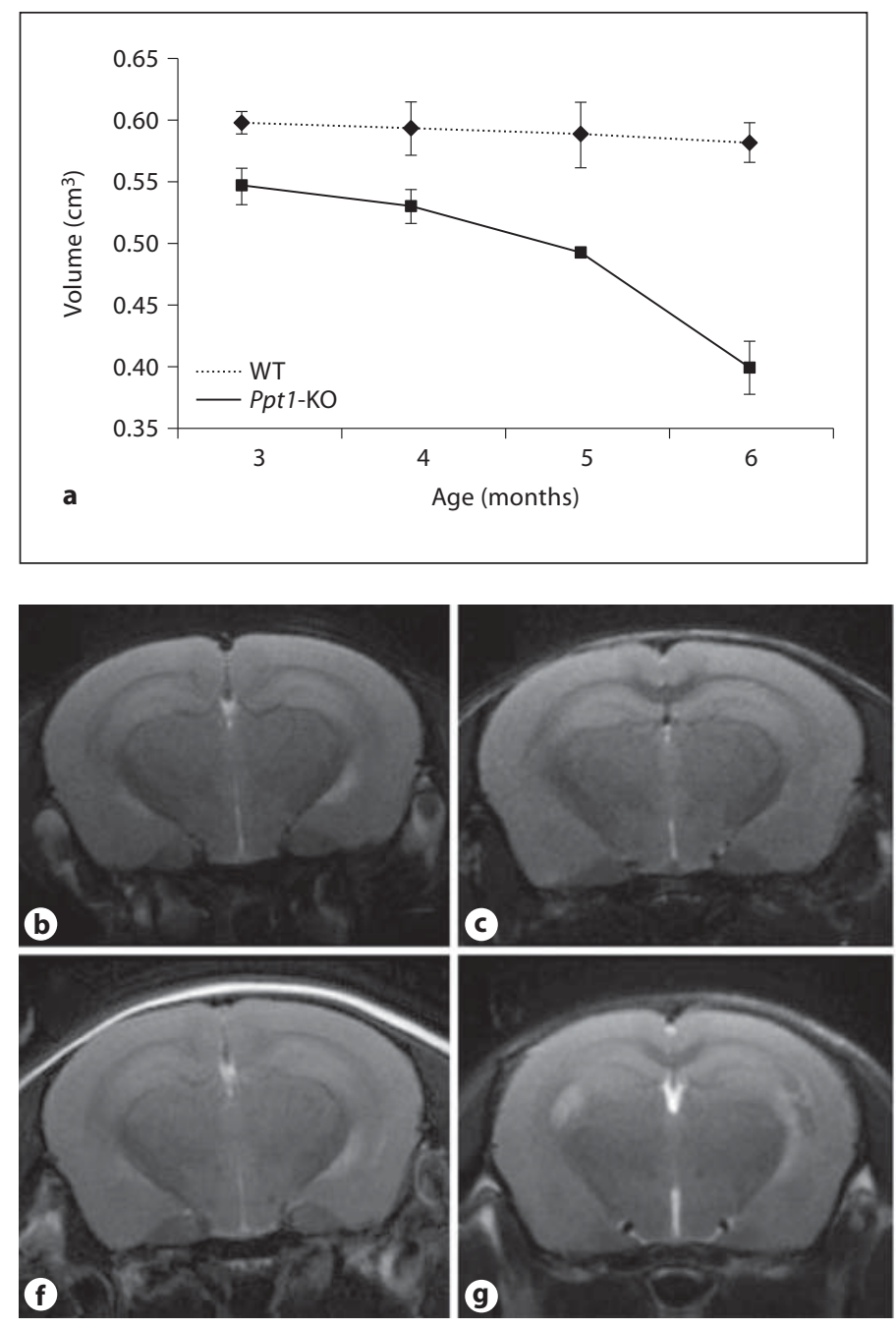

Fig. 1. Evaluation of brain volume and size in WT and Ppt1-KO mice by MRI. Ppt1-KO mice and their WT littermates were first evaluated at 3 months of age by MRI. These evaluations were again repeated when these mice were 4, 5 and 6 months of age. The brain volumes were calculated from the MRI as described in 'Animals and Methods' and average brain volumes are plotted (a). The results are the means $(n=3 \pm S D) . T_{2}$-weighted axial images of the WT mice (b-e) and their Ppt1-KO littermates at corresponding ages $(\mathbf{f}-\mathbf{i})$. Note the progression of neurodegeneration in the Ppt1-KO mice, which is readily detectable from as early as 3 months of age. The decline in brain volumes (a) positively correlates with smaller brain sizes of Ppt1-KO mice, especially with increasing age.

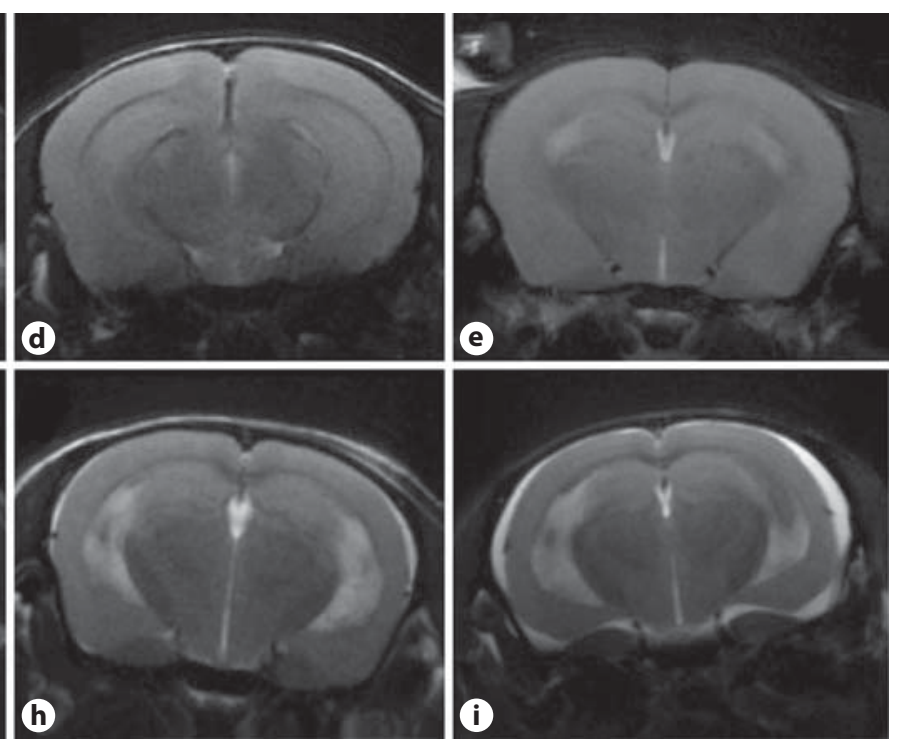

clinical and pathological features of INCL [28], including reduced volume of cortical and subcortical regions of the brain and sparing of the cerebellum [29]. Thus, we sought to determine whether brain volumes calculated from MR images can be used to noninvasively evaluate the progression of brain degeneration in Ppt1-KO mice. Accordingly, we performed brain MRI of WT mice and their Ppt1-KO littermates at 3 months of age and repeated the procedure at 4, 5 and 6 months of age. The calculated brain volumes (in $\mathrm{cm}^{3}$ ) of WT versus Ppt1-KO mice were as follows: 3 months of age $=\mathrm{WT}, 0.597 \pm 0.008$ versus $\mathrm{KO}, 0.546 \pm$ $0.015 ; 4$ months of age $=\mathrm{WT}, 0.593 \pm 0.022$ versus $\mathrm{KO}$, $0.53 \pm 0.014 ; 5$ months of age $=\mathrm{WT}, 0.588 \pm 0.027$ versus $\mathrm{KO}, 0.493 \pm 0.004$; and 6 months of age $=\mathrm{WT}, 0.582 \pm$ 0.016 versus $\mathrm{KO}, 0.399 \pm 0.022$.

The results showed that $\mathrm{T}_{2}$ times of weighted images clearly demonstrated that compared with WT mice (fig. 1b-e) the Ppt1-KO littermates (fig. 1f-i) undergo progressive and significant reductions in brain volume. Although qualitative image analysis did not show discernible visual changes, the differences in brain volumes of Ppt1-KO mice and their WT littermates (calculated using the $\mathrm{T}_{2}$-weighted images) were clearly appreciable at 3 months of age (fig. 1a). These differences became markedly conspicuous from 4 months of age (fig. 1a), which progressed further to significant atrophy around 6 months of age. Due to the marked atrophy of the brain in Ppt1-KO mice, accumulation of extracerebral fluid collection was visualized as hyperintense areas of the brain of these mice at 6 months of age (fig. 1i). Consistent with these observations, similar findings were recently reported in INCL patients [30]. In addition to the reduction in cortical volumes, the size of lateral ventricles, especially of 6-month-old Ppt1-KO mice, was appreciably enlarged. 


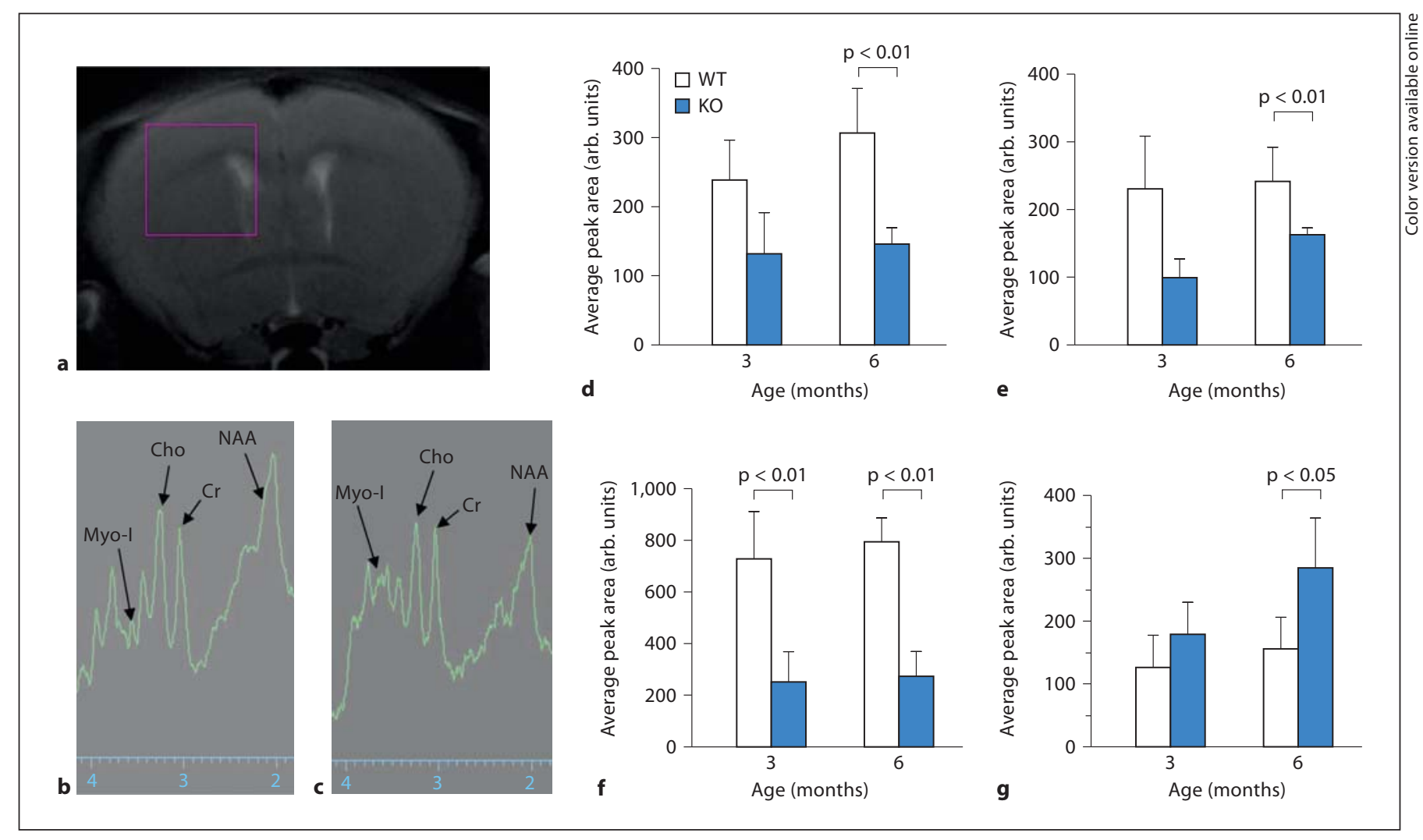

Fig. 2. Neurodegeneration in Ppt1-KO mice measured by single voxel MRS. MRS of a $2.5 \mathrm{~mm}^{3}$ single volume element (voxel) selected using a pilot MR image which encompassed part of the cortex, the hippocampus and ventricles (a). MR spectra from the chemical shift range of a representative WT (b) and Ppt1-KO (c) mouse showing selected peaks of interest, creatine (Cr), choline (Cho), NAA and myoinositol (Myo-I). Average areas under the MR peaks of the metabolites creatine (d), choline (e), NAA (f) and myoinositol (g) for WT and Ppt1-KO are shown.
However, in this study, we did not make any attempt to evaluate the changes in ventricular size. Taken together, our results show a rapidly progressive decline in cortical volume, which is detectable as early as 3 months of age of these mice. These results are consistent with neurodegeneration beginning at an early age and continuing over time, as previously reported in patients with INCL $[3,4]$.

\section{MR Spectral Analysis}

Decline in several brain chemicals including NAA is a reliable measure of neurodegeneration [31, 32]. Accordingly, we monitored several brain chemicals in Ppt1-KO mice from MR spectra (fig. 2a) and calculated their relative concentrations. The results of our analysis of the spectra from selected brain chemicals and metabolites (fig. $2 \mathrm{~b}$ and c, arrows) allow us to make several conclusions. First, there was a significant reduction in creatine (fig. 2d) in the brains of 6-month-old Ppt1-KO mice com- pared with those of their WT littermates ( $p<0.01$ for 6-month-old Ppt1-KO vs. WT mice). Second, the brain choline levels declined significantly (fig. 2e), suggesting the possible loss of vital membrane and myelin constituents ( $\mathrm{p}<0.01$ for 6 -month old Ppt1-KO vs. WT mice). The reduction in choline levels in Ppt1-KO mice as early as 3 months of age may be an early indication of the onset of neuronal death, most likely by apoptosis, which has been previously reported to increase with advancing age in these mice [33-36]. Third, compared with their WT littermates, the brains of Ppt1-KO mice contained appreciably lower levels of the neuronal marker NAA at all age groups (fig. 3f), indicating that there was significant loss of neurons in the diseased mice even at the lowest agegroup studied ( $\mathrm{p}<0.01$ for 3 -month-old Ppt1-KO vs. WT, and $\mathrm{p}<0.01$ for 6 -month-old $P p t 1-\mathrm{KO}$ vs. WT mice). Finally, the elevated levels of myoinositol (fig. $2 \mathrm{~g}$ ) in the brain of 6-month-old Ppt1-KO mice ( $\mathrm{p}<0.05$ for 6-month 
Fig. 3. Variation of ADC and spin-spin relaxation $\left(\mathrm{T}_{2}\right)$ times. Quantitative ADC (a) and $\mathrm{T}_{2}$ values (b), evaluated from averaging over ten selected regions $\left(0.4 \mathrm{~mm}^{2}\right)$ of interest in the cortical region for 3-monthold and 6-month-old WT mice and Ppt1KO littermates. Calculated ADC values highlight inherent tissue characteristics as they accentuate possible early changes in water diffusion, due to changes in the microscopic environment, upon the onset of the disease that manifests as increased $\mathrm{ADC}$ as early as 3 months of age of the Ppt1-KO mice. In contrast, $\mathrm{T}_{2}$ which reflects coherence and molecular interactions within this environment shows no significant change.

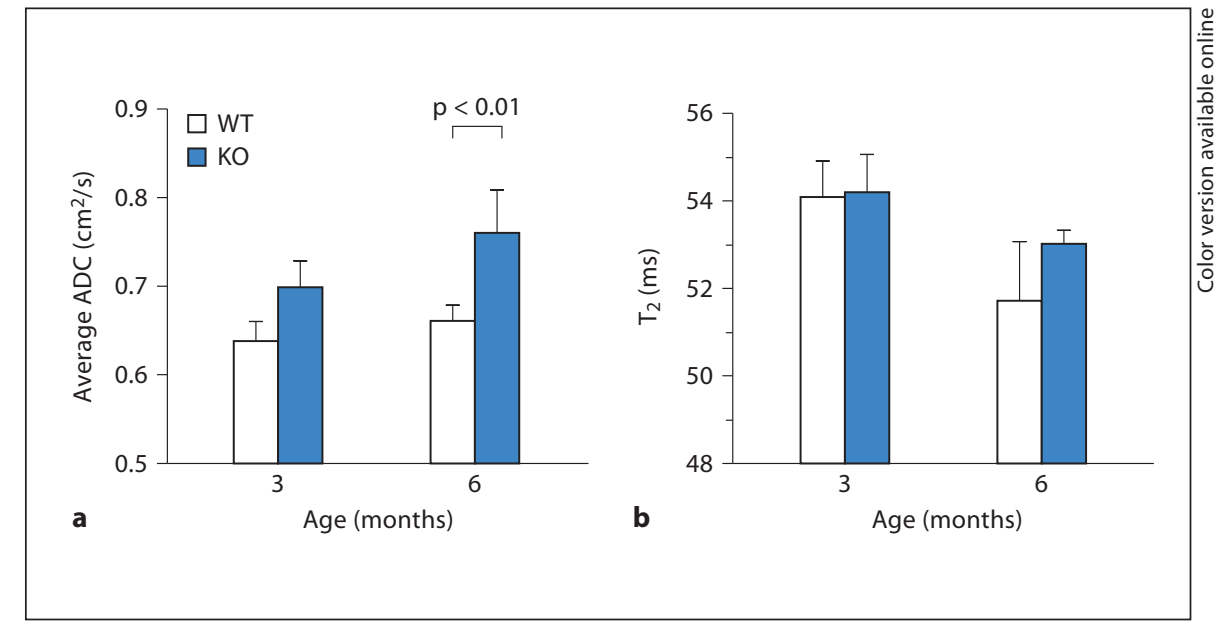

old Ppt1-KO vs. WT mice) suggested a greater presence of activated astroglial cells, which infiltrate the brain most likely to scavenge the apoptotic neurons [37]. These results suggest a pattern of transition from a predominantly neuronal population to activated astroglia with age in Ppt1-KO mice.

\section{$A D C$ and $T_{2}$ Measurements}

Diffusion of water in the heterogeneous brain tissue can be probed by DW-MRI. DW-MRI yields in vivo images of brain tissues that depict the diffusion of water kindred to the local microstructural characteristics. In DW imaging, each image voxel (a 3D volume element) has an image intensity that reflects the single best measurement of the rate of water diffusion at that location. The apparent water diffusion coefficients can be calculated by acquiring two or more images with a different gradient, duration and amplitude. Thus, ADC measures the magnitude of diffusion, while the contrast in the ADC images depends on the spatially distributed diffusion coefficient of the tissues. The facilitation or hindrance to diffusion of water within the microenvironment of the brain tissue is reflective of the ADC maps. The average ADC maps (from three different orthogonal directions) render voxel-by-voxel information into microstructural integrity of the tissue. Thus, we measured the ADCs of the cerebral cortex in Ppt1-KO mice and those of their WT littermates. The calculated ADC values for WT versus $P$ pt1-KO mice were: 3 -month-old WT versus $P$ pt1-KO, $0.64 \pm 0.02$ versus $0.70 \pm 0.03 \mathrm{~cm}^{2} / \mathrm{s}$; and 6-month-old WT versus $P$ pt1-KO, $0.60 \pm 0.02$ versus $0.76 \pm 0.05 \mathrm{~cm}^{2} / \mathrm{s}$. These results show a significant increase in cortical ADC values (fig. 3a) in Ppt1-KO mice $(p<0.009)$, which increased with age. As expected, the ADCs in WT mice did not increase with age $(\mathrm{p}<0.4)$ as previously reported [38]. The differences in ADC values were significant $(\mathrm{p}<0.007)$ between WT and Ppt1-KO mice as early as 3 months of age. The calculated $\mathrm{T}_{2}$ values for WT versus $P$ pt1-KO mice were: 3 -month-old WT versus $P$ pt1-KO, $54.08 \pm 0.81$ versus $54.15 \pm 0.91 \mathrm{~ms}$; and 6-month-old WT versus $P$ pt1-KO, $51.73 \pm 1.30$ versus $52.99 \pm 0.35 \mathrm{~ms}$. In contrast to ADC values, no significant differences were observed when $\mathrm{T}_{2}$ values from cerebral cortical regions of WT mice were compared with those of their Ppt1-KO counterparts at 3 months ( $\mathrm{p}<$ $0.49)$ and 6 months of age ( $<<0.76$; fig. $3 b)$. Taken together these results suggest that in the Ppt1-KO mouse brain, ADCs are markedly higher than in those of their WT littermates, indicating alteration in the brain microenvironment and disruption of brain tissue integrity in these mice.

Progressive Loss of Neurons and Increased Astroglia in the Brain of Aging Ppt1-KO Mice

The results of MRI and MRS analysis described above suggest a loss of brain volume, decreased neuronal marker and increased astroglial marker in the Ppt1-KO mouse brain. However, it is not clear whether these MRI and MRS findings correlate with actual loss of neurons and increased astroglia. Accordingly, we carried out immunohistological analyses of the brain sections from 3-, 4-, 5- and 6-month-old Ppt1-KO mice and those of their WT littermates using Golgi-Cox staining (for detecting neurons) and glial fibrillary acidic protein (for astroglia), respectively. Our results show that while a comparison of neuronal density (neurons $/ \mathrm{mm}^{2}$ ) in the brain of 3-month- 


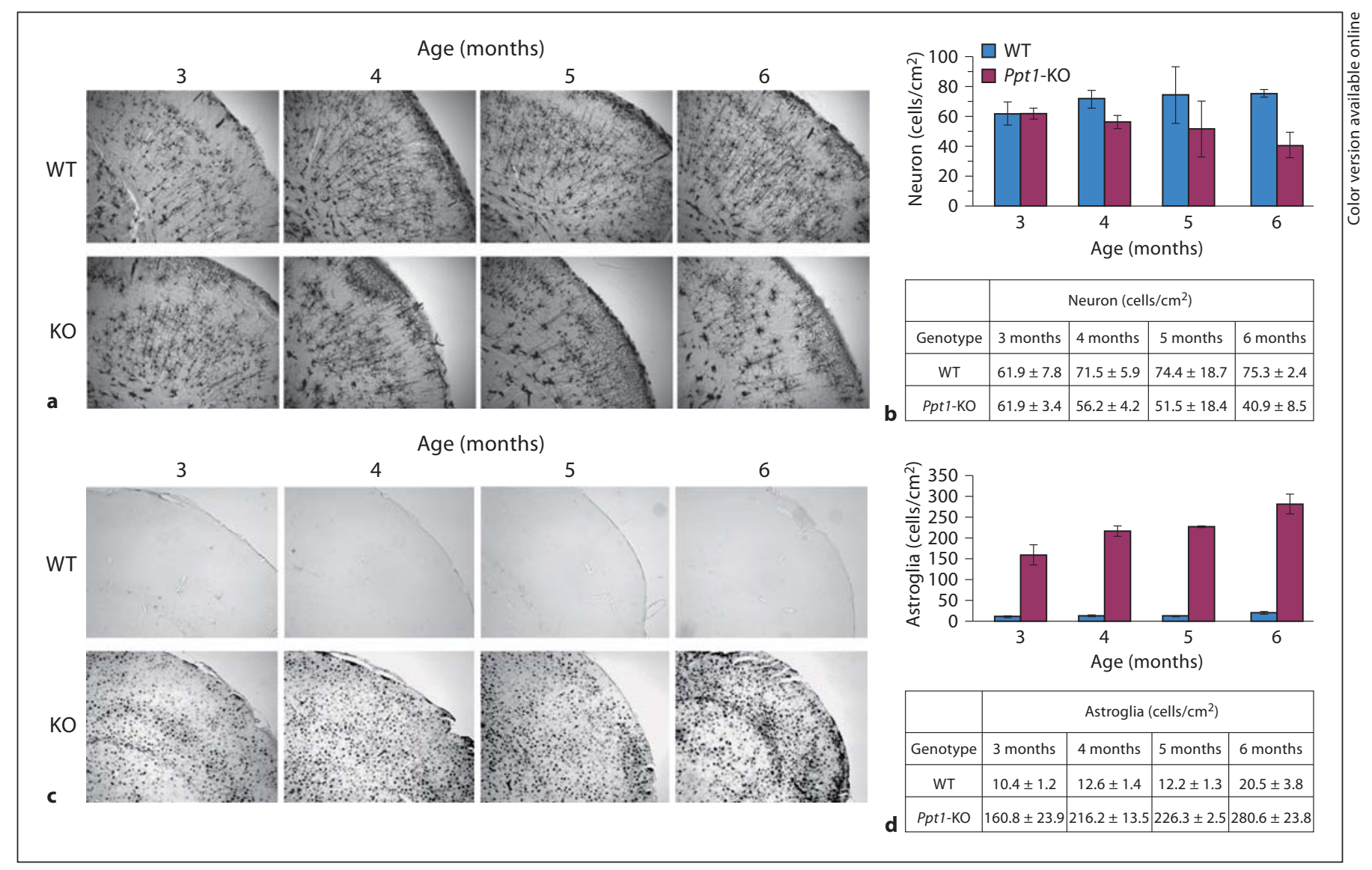

Fig. 4. Immunohistopathology of brain sections from Ppt1-KO mice and WT littermates. Numbers of neurons and levels of glial fibrillary acidic protein in the brains of Ppt1-KO and WT littermates were determined. a Detection of neuronal cells in Ppt1-KO and WT littermates. Cortical neurons from Ppt1-KO and WT mice (3-, 4-, 5- and 6-month-old) were visualized by the GolgiCox staining. b Quantification of the neurons. Three different areas of the cortical tissue were picked randomly, and the cell bod- ies were countered. Stand deviation was calculated and Student's $t$ test was used to determine the significant difference. c Immunohistochemical detection of astroglial cells in Ppt1-KO and WT littermates. d Quantitation of the astroglial cells. Three different areas of the cortical tissue were randomly chosen to determine the number of astroglia $/ \mathrm{mm}^{2}$ area. The results are expressed as the means of 3 determinations $\pm \mathrm{SD}$.

\section{Changes in rCBV in Ppt1-KO Mouse Brain}

Cerebral perfusion maintains the hemodynamic demands of microscopic environments in the brain. Variation in cerebral blood flow and $\mathrm{rCBV}$ signify changes in brain perfusion. Thus, rCBV estimates the fraction of tissue volume occupied by blood vessels. The contrast agent USPIO remains intravascular and produces hypointense signals in MRI, which can be related to rCBV. The brain perfusion in atrophic brains may show differences, which are reflective of rCBV images. The results of our calculations show a significant increase in rCBV of 6-month-old Ppt1-KO mice compared with that of their WT littermates ( $\mathrm{p}<0.0003$; fig. 5a, $\mathrm{c}, \mathrm{e})$. Interestingly, the differences in rCBVs between 3-month-old Ppt1-KO mice and 


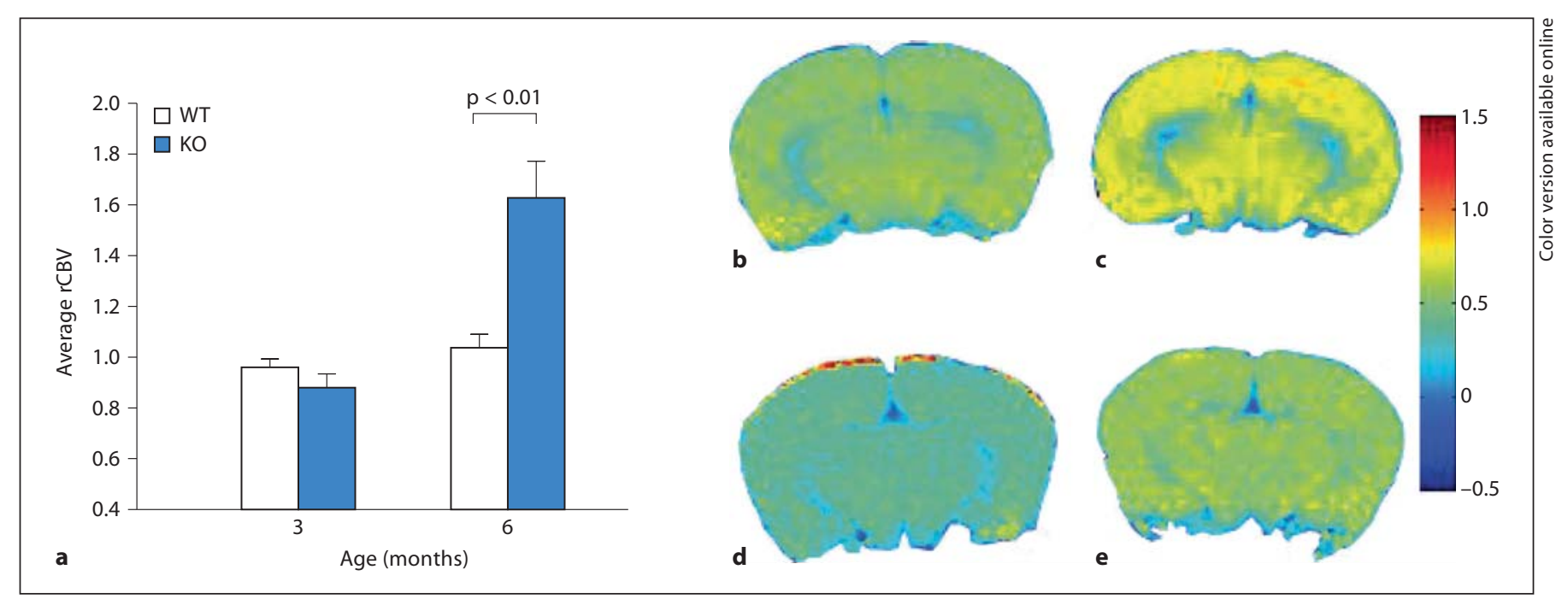

Fig. 5. Variation of $\mathrm{rCBV}$ with age. a Plot of average percentage rCBV variation, calculated over ten selected $0.4-\mathrm{mm}^{2}$ regions of interest in the cortical regions of WT mice and those of their Ppt1KO littermates. Corresponding rCBV map between 3-month-old Ppt1-KO (b) and 6-month-old Ppt1-KO (c) mice show increased

that of their WT littermates are not significant $(\mathrm{p}<0.51$; fig. 5b, d). These results may signify changes in vascular integrity, which progressively gets worse in the Ppt1-KO mice with age.

\section{Discussion}

In this study, we demonstrated that neurodegeneration in Ppt1-KO mice can be repeatedly evaluated by combining MRI and MRS analyses. Brain volume changes were detectable as early as 3 months of age in the Ppt1$\mathrm{KO}$ mice and these changes progressed with increasing age as observed in INCL patients [30]. Elevated ADC values in Ppt1-KO mice suggested alteration in the brain microenvironment and disruption of neuronal integrity in these mice. While we did not measure the ventricular volumes in the Ppt1-KO mice, the apparent increase in size of the ventricles compared to WT littermates was visually appreciable. The MRS analyses showed a generalized decrease in NAA, a biochemical marker for the neurons, in the Ppt1-KO mice. The progressive increase in myoinositol was most likely due to increased infiltration of activated astroglial cells.

The metabolic changes uncovered in this study was considered to be from the gray matter where most significant degenerative changes occur in INCL, assuming changes in microvasculature in the latter due to the magnetic susceptibility contrast, induced by the presence of USPIO. In contrast, the maps for 3-month old (d) and 6-month old WT mice (e) show relatively low rCBV changes similar to 3 month-old Ppt1$\mathrm{KO}$ mice (b).

that any alterations from the white matter and cerebrospinal fluid were minimal. The decline in the levels of NAA indicated a reduction of the neuronal population and an increase in myoinositol levels suggested infiltration of astroglial cells in the Ppt1-KO mouse brain. Interestingly, the NAA levels in the brains of Ppt1-KO mice of all age groups were significantly lower than those of their WT littermates, which might be the reason why a progressive decline in mice of 3-6 months of age was not appreciable. Thus, it appears that the onset of neuronal degeneration occurs earlier than 3 months of age. The other likely possibility may be that due to the progressive decline in cortical volume, voxel size used in this study covered only a fraction of the ventricle and the hippocampus as the brain volume declined. Therefore, the NAA concentration for a given voxel is likely to have a higher variability in these mice. Thus, although we assume a significant reduction in NAA occurs with disease progression, it is possible that curve fitting overestimates the relative peak area of NAA. This problem may be rectified by using voxels covering a smaller area in the MRS to include only the cortex. However, this approach may impose other experimental constraints. First, the naturally low metabolite concentration that gives rise to relatively weak MR spectral peaks may be diminished significantly when the detection volume (voxel size) is reduced, thus leading to even weaker MR spectra. Second, 
reduction in voxel size would impose stricter constraints on the hardware that would be necessary to achieve smaller voxels. One alternative approach may be to acquire the MRS repeatedly to increase the metabolite signal intensity. However, this may add considerable amount of time to in vivo scanning, which will require prolonged anesthesia that may compromise animal survival. While further studies are needed to improve these parameters, one caveat that needs to be addressed is the increased length of time required to complete these studies, which may alter the physiology of the brain of these animals under anesthesia for a prolonged period of time.

To confirm that the decreased NAA and increased myoinositol levels indicate decreased number of neurons and increased astroglia, respectively, we performed immunohistochemical analysis of the brains from age- and sex-matched Ppt1-KO mice and those of their WT littermates. We analyzed the brain sections using Golgi-Cox staining for neurons and antibodies against glial fibrillary acidic protein for astroglia. The results of immunohistochemical analyses correlated well with those of the MRI/MRS studies. A detailed histochemical study on neurodegeneration in Ppt1-KO mice was conducted and the results of this study showed successive neuron loss in the thalamus and cortex of these mice [28]. Our MRI and MRS results on live Ppt1-KO mice are consistent with the results described in this study. Thus, the progressive decline in brain volume quantitated from brain MRI appears to indicate a loss of neuronal population in the brain of Ppt1-KO mice with corresponding increase in a glial fibrillary acidic protein staining, which is indicative of the astroglial population. The later finding may also suggest the presence of neuroinflammation as increased astroglia in the brain of the Ppt1-KO mice has been reported to increase with this condition [37].

ADC portrays the heterogeneity of molecular diffusion effects of water within the brain microenvironment $[38,39]$. These results suggest that the Ppt1-KO mouse brains undergo structural changes which allow increased degrees of freedom for the diffusion of water to take place at a very early stage. These could be due to a cascade of events from reorganization or breakdown of cellular architecture, which in-turn could manifest as increase in ADC. $T_{2}$ relaxation time maps characterize how fast excited water magnetization returns to equilibrium. In the brain, $\mathrm{T}_{2}$ variations are also related to the interaction of water within cellular/tissue components in its microenvironment. The decrease in $\mathrm{T}_{2}$ with age could be due to the variation in the degree of fiber myelination [40]. However, unlike ADC, the local interactions, which may change relaxation rate differences, do not seem to be affected with the disease. Similar observations have been reported for other insults such as cerebral ischemia [41].

$\mathrm{rCBV}$ is an important cerebrovascular parameter. USPIO, the paramagnetic intravascular contrast agent, has a relatively long half-life in the blood. Once infused, USPIO remains intravascular and the magnetic susceptibility of iron induces increased $\mathrm{T}_{2}$ relaxation [42]. This leads to hypointense regions in MR images depicting quantitative information of microvasculature in specific neural tissues which eventually may be coupled to the physiologic state of the brain. The magnetic susceptibility-induced contrast in WT mice and their 3-month-old Ppt1-KO littermates reflects rCBV. However, the increase in rCBV in the brain of 6-month-old Ppt1-KO mice, whose microvasculature may be rendered leaky [43] due to neurodegeneration and, therefore, the calculated $\mathrm{rCBV}$ values) are likely to be influenced by the interplay of altered vessel properties. Thus, the increased rCBV measured in 6-month-old Ppt1-KO mice is likely to be slightly overestimated due to the magnetic susceptibility effects as USPIO diffuse to the intracellular spaces. Furthermore, neurological damage which may lead to hypoxia can lead to vasodilation of the cerebral microvessels and show increased rCBV values [44], which is likely in 6-month-old Ppt1-KO mice. These mice may also have reduced cerebral compliance, and hypoxia-induced vasodilation can impair function of more vessels leading to increased USPIO leakage to the tissues. As neuronal apoptosis is prevalent in the brain of these mice [33-36], angiogenesis, and possible increase in rCBV [45], may alter the level of tissue perfusion affecting viability of neural tissues. One other possibility for increased rCBV could be due to engulfment of USPIO by phagocytes, which infiltrates the brain of these mice [46]. However, since our USPIO scans are completed within 5 min of infusion, it is highly unlikely that astroglial cells (phagocytes) can engulf a significant amount of USPIO within this short time to affect rCBV.

To our knowledge, this is the first time an attempt has been made to repeatedly evaluate and document the progressive nature of neurodegeneration in an animal model of INCL without sacrificing the animals. In this study, the brain volume measurements appear to be the most reliable method of evaluating neurodegeneration, although the use of smaller voxel for MRS may also yield reliable results. Thus, our results suggest that MRI and MRS are useful tools in evaluating the effectiveness of novel therapeutic interventions in this animal model of INCL, a devastating hereditary neurodegenerative lysosomal storage disease. 


\section{Acknowledgements}

We thank S.L. Hofmann for the generous gift of the Ppt1-KO mice and for helpful comments and suggestions on the manuscript. We also thank J.Y. Chou, I. Owens and S.W. Levin for critical review of the manuscript and helpful suggestions. We are grateful to Ms. Mary Angstadt of the MRI center, NINDS, for help with preparation of the animals for MRI/MRS. This research was supported in part by the Intramural Research Program of the Eunice Kennedy Shriver National Institute of Child Health and Human Development, National Institutes of Health, and The Batten Disease Support and Research Association (BDSRA).

\section{References}

1 Proia RL, Wu YP: Blood to brain to the rescue. J Clin Invest 2004;113:1108-1110.

2 Sly WS, Vogler C: Brain-directed gene therapy for lysosomal storage disease: going well beyond the blood-brain barrier. Proc Natl Acad Sci USA 2002;99:5760-5762.

3 Goebel HH, Wisniewski KE: Current state of clinical and morphological features in human NCL. Brain Pathol 2004;14:61-69.

4 Haltia M: The neuronal ceroid-lipofuscinoses: from past to present. Biochim Biophys Acta 2006; 1762:850-856.

$>5$ Hobert JA, Dawson G: Neuronal ceroid lipo- 17 fuscinoses therapeutic strategies: past, present and future. Biochim Biophys Acta 2006; 1762:945-953.

6 Hofmann SL, Peltonen L: The neuronal ceroid lipofuscinoses; in Scriver CR, et al (eds): The Metabolic and Molecular Bases of Inherited Disease, ed 8. New York, McGrawHill, 2001, pp 3877-3894.

7 Jalanko A, Braulke T: Neuronal ceroid lipofuscinoses. Biochim Biophys Acta 2009; 1793:697-709.

8 Mitchison HM, Mole SE: Neurodegenerative disease: the neuronal ceroid lipofuscinoses (Batten disease). Curr Opin Neurol 2001;14: 795-803.

9 Mole SE: The genetic spectrum of human neuronal ceroid-lipofuscinoses. Brain Pathol 2004;14:70-76.

-10 Siintola E, Lehesjoki AE, Mole SE: Molecular genetics of the NCLs - status and perspectives. Biochim Biophys Acta 2006;1762:857864.

11 Cooper JD, Russell C, Mitchison HM: Progress towards understanding disease mechanisms in small vertebrate models of neuronal ceroid lipofuscinosis. Biochim Biophys Acta 2006; 1762:873-889.

12 Resh M: Palmitoylation of ligands, receptors, and intracellular signaling molecules. Sci STKE 2006;2006:re14.

$\checkmark 13$ Smotrys JE, Linder ME: Palmitoylation of intracellular signaling proteins: regulation and function. Annu Rev Biochem 2004;73: 559-587.

14 Linder ME, Deschenes RJ: Palmitoylation: policing protein stability and traffic. Nat Rev Mol Cell Biol 2007;8:74-84.
15 Kang R, Wan J, Arstikaitis P, Takahashi H, Huang K, Bailey AO, Thompson JX, Roth AF, Drisdel RC, Mastro R, Green WN, Yates JR 3rd, Davis NG, El-Husseini A: Neural palmitoyl-proteomics reveals dynamic synaptic palmitoylation. Nature 2008;456:904-909.

16 Lu JY, Verkruyse LA, Hofmann SL: Lipid thioesters derived from acylated proteins accumulate in infantile neuronal ceroid lipofuscinosis: correction of the defect by recombinant palmitoyl-protein thioesterase. Proc Natl Acad Sci USA 1996;93:10046-10050.

17 Duncan JA, Gilman AG: A cytoplasmic acylprotein thioesterase that removes palmitate from $G$ protein alpha subunits and p21RAS. J Biol Chem 1998;273:15830-15837.

18 Tomatis VM, Trenchi A, Gomez GA, Daniotti JL: Acyl-protein thioesterase 2 catalyzes the deacylation of peripheral membrane-associated GAP-43. PLoS One 2010; 5:e15045.

19 Camp LA, Hofmann SL: Purification and properties of a palmitoyl-protein thioesterase that cleaves palmitate from H-Ras. J Biol Chem 1993:268:22566-22574.

20 Camp LA, Verkruyse LA, Afendis SJ, Slaughter CA, Hofmann SL: Molecular cloning and expression of palmitoyl-protein thioesterase. J Biol Chem 1994:269:23212-23219.

-21 Gupta P, Soyombo AA, Atashband A, Wisniewski KE, Shelton JM, Richardson JA, Hammer RE, Hofmann SL: Disruption of PPT1 or PPT2 causes ceriod lipofuscinosis in knockout mice. Proc Natl Acad Sci USA 2001;98:13566-1357.

22 Verkruyse LA, Hofmann SL: Lysosomal targeting of palmitoyl-protein thioesterase. J Biol Chem 1996;271:15831-15836.

23 Hellsten E, Vesa J, Olkkonen VM, Jalanko A Peltonen L: Human palmitoyl protein thioesterase: evidence for lysosomal targeting of the enzyme and disturbed cellular routing in infantile neuronal ceroid lipofuscinosis. EMBO J 1996;15:5240-5245.

24 Baekkeskov S, Kanaani J: Palmitoylation cycles and regulation of protein function (review). Mol Membr Biol 2009;26:42-54.

25 Salaun C, Greaves J, Chamberlain LH: The intracellular dynamic of protein palmitoylation. J Cell Biol 2010;191:1229-1238.

26 Vesa J, Hellsten E, Verkruvse LA, Camp LA, Rapola J, Santavuori P, Hofmann SL, Peltonen L: Mutations in the palmitoyl protein thioesterase gene causing infantile neuronal ceroid lipofuscinosis. Nature 1995;376:584-587.
27 Gupta P, Soyombo AA, Atashband A, Wisniewski KE, Shelton JM, Richardson JA, Hammer RE, Hofmann SL: Disruption of PPT1 and PPT2 causes neuronal ceroid lipofuscinosis in knockout mice. Proc Natl Acad Sci USA 2001;98:13566-13571.

28 Bible E, Gupta P, Hofmann SL, Cooper JD: Regional and cellular neuropathology in the palmitoyl protein thioesterase-1 null mutant mouse model of infantile neuronal ceroid lipofuscinoses. Neurobiol Dis 2004; 16:346359.

29 Kielar C, Maddox L, Bible E, Pontikis CC, Macauley SL, Griffey MA, Wong M, Sands MS, Cooper JD: Successive neuron loss in the thalamus and cortex in a mouse model of infantile neuronal ceroid lipofuscinosis. Neurobiol Dis 2007;25:150-162.

30 Levin SW, Baker EH., Gropman A, Quezado Z, Miao N, Zhang Z, Jollands A, Di Capua M, Caruso R, Mukherjee AB: Subdural fluid collections in patients with infantile neuronal ceroid lipofuscinoses. Arch Neurol 2009; 66:1567-1571.

- 31 Erichsen AK, Server A, Landrø NI, Sandvik L, Tallaksen CM: Proton magnetic resonance spectroscopy and cognition with spastin mutations. J Neurol Sci 2009;277:124129.

32 Tkáč I, Starčuk Z, Choi IY, Gruetter R: In vivo $1 \mathrm{H}$ NMR spectroscopy of rat brain at 1 ms echo time. Magn Reson Med 1999;41: 649-656.

33 Kim SJ, Zhang ZJ, Hitomi E, Lee YC, Mukherjee AB: Endoplasmic reticulum stress-induced caspase- 4 activation mediates apoptosis and neurodegeneration in INCL. Hum Mol Genet 2006;15:1826-1834.

34 Zhang Z, Lee YC, Kim SJ, Choi MS, Tsai PC, Xu Y, Xiao YJ, Zhang P, Heffer A, Mukherjee AB: Palmitoyl-protein thioesterase-1 deficiency mediates the activation of the unfolded protein response and neuronal apoptosis in INCL. Hum Mol Genet 2006;15:337-346.

-35 Wei H, Kim SJ, Zhang Z, Tsai PC, Wisniewski KE, Mukherjee AB: ER- and oxidativestresses are common mediators of apoptosis in both neurodegenerative and non-neurodegenerative lysosomal storage disorders and are alleviated by chemical chaperones. Hum Mol Genet 2008;17:469-477. 
36 Wei H, Zhang Z, Saha A, Peng S, Chandra G, Quezado Z, Mukherjee AB: Disruption of adaptive energy metabolism and elevated ribosomal p-S6K1 levels contribute to INCL pathogenesis: partial rescue by resveratrol. Hum Mol Genet 2011;20:1111-1121.

- 37 Zhang Z, Lee YC, Kim SJ, Choi MS, Tsai PC, Saha A, Wei H, Xu Y, Xiao YJ, Zhang P, Heffer A, Mukherjee AB: Production of lysophosphatidylcholine by cPLA2 in the brain of mice lacking PPT1 is a signal for phagocyte infiltration. Hum Mol Genet 2007;16: 837-847.

38 Rau PR, Sellner J, Heiland S, Plaschke K, Schellinger PD, Meyding-Lamadé UK, Lamadé WR: Apparent diffusion coefficient in the aging mouse brain: a magnetic resonance imaging study. Life Sci 2006;78:1175-1180.
39 Le Bihan D: Looking into the functional architecture of the brain with diffusion MRI. Nat Rev Neurosci 2003;4:469-480.

40 Guilfoyle DN, Dyakin VV, O'Shea J, Pell GS, Helpern JA: Quantitative measurements of proton spin-lattice (T1) and spin-spin (T2) relaxation times in the mouse brain at 7.0 T. Magn Reson Med 2003;49:576-580.

41 Mintorovitch J, Moseley ME, Chileuitt L, Shimizu H, Cohen Y, Weinstein PR: Comparison of diffusion- and T2-weighted MRI for the early detection of cerebral ischemia and reperfusion in rats. Magn Reson Med 1991;18:39-50.

42 Wu EX, Wong KK, Andrassy M, Tang H: High-resolution in vivo CBV mapping with MRI in wild-type mice. Magn Reson Med 2003;49:765-770.

-43 Blamire AM, Anthony DC, Rajagopalan B, Sibson NR, Perry VH, Styles P: D2 interleukin-1beta-induced changes in blood-brain barrier permeability, apparent diffusion coefficient, and cerebral blood volume in the rat brain: a magnetic resonance study. J Neurosci 2000;20:8153-8159.
44 Julien-Dolbec C, Tropres I, Montigon O, Reutenauer H, Ziegler A, Decorps M, Payen JF: Regional response of cerebral blood volume to graded hypoxic hypoxia in rat brain. Br J Anaesthesia 2002;89:287-293.

-45 Swain RA, Harris AB, Wiener EC, Dutka MA, Morris HD, Theien BE, Konda S, Engberg K, Lauterbur PC, Greenouh WT: Prolonged exercise induces angiogenesis and increases cerebral blood volume in primary motor cortex of the rat. Neuroscience 2003; 117:1037-1046

46 Corot C, Robert P, Idée J-M, Port M: Recent advances in iron oxide nanocrystal technology for medical imaging. Ad Drug Deliv Rev 2006;58:1471-1504. 